\title{
Análisis biomecánico de la ejecución técnica del Toss to hands en el Cheerleaders.
}

\author{
Biomechanical analysis of the technical execution of Toss to hands in \\ Cheerleading.
}

Oscar Andrés Orbe Yumisaca. ${ }^{1}$

\begin{abstract}
DOI: https://doi.org/10.33262/cienciadigital.v5i3.1731

Introduction. The optimization of performance in any sport discipline, as in the case of cheerleaders, depends on their execution biomechanics. The Toss to hands technique is an action in pairs with biomechanically similar base and flayer roles. Objetive. Build a structural technical model of the execution of the Toss to hands technique in cheerleaders, taking into account the roles that each athlete performs and the characterization of the biomechanical parameters of angles in different joints, speeds and maximum heights in each determined phase. Methodology. The study was based on a mixed descriptive approach with a cross section, supported by the use of theoretical and empirical methods. Results. 1. The documentary and observational investigation of the videos made to the sample under study, made up of 25 athletes between bases and flayers, allowed to build a structural technical model of the technical execution of the Toss to hands in the cheerleaders, referring to components, periods, phases and movement actions, which are described and differentiated in each role. 2 . It could be seen that the angles of the elbow, hip and knee joints differ in each role with significant differences in each phase of movement, as well as the speed of execution, which presents higher values in the projection and dismount phases by part of the flayer, the analysis of the maximum heights similarly presented significant differences, evidenced by the application of the one-factor ANOVA statistical test. Conclution. A structural technical model was built in phases, describing 3 components, 3 periods and 11 phases for each role, which turns out to be
\end{abstract}

\footnotetext{
${ }^{1}$ Maestrante del programa de entrenamiento deportivo de la Universidad Central del Ecuador, Facultad de Cultura Física, oaorbe@uce.edu,ec
} 
more comprehensive, allowing to target the optimization of sports performance and the improvement of both individual and pair competition results.

Keywords: Toss to hands, cheerleaders, phases of movement, base, flayer.

\section{Resumen}

Introducción. La optimización del rendimiento en cualquier disciplina deportiva, como en el caso del cheerleaderes, dependen de su biomecánica de ejecución. La técnica del Toss to hands es una acción en parejas con roles de base y flayer, biomecánicamente similares. Objetivo. Construir un modelo técnico estructural de la ejecución de la técnica del Toss to hands en el cheerleaders, tomando en cuenta los roles que cada atleta ejecuta y la caracterización de los parámetros biomecánicos de ángulos en diferentes articulaciones, velocidades y alturas máximas en cada fase determinada. Metodología. El estudio se basó en un enfoque mixto de tipo descriptivo con un corte transversal, apoyado en el empleo de métodos teóricos y empíricos. Resultados. 1. La investigación documental y observacional de los videos realizados a la muestra objeto de estudio integrada por 25 atletas entre bases y flayers, permitió construir un modelo técnico estructural de la ejecución técnica del Toss to hands en el cheerleadres, refiriendo componentes, periodos, fases y acciones de movimiento, que se describen y diferencian en cada rol. 2. Se pudo apreciar que los ángulos de las articulaciones del codo, cadera y rodilla se diferencian en cada rol con diferencias significativas en cada fase de movimiento, al igual que la velocidad de ejecución que presenta valores mayores en las fases de proyección y desmonte por parte del flayer, el análisis de las alturas máximas de igual manera presentó diferencias significativas, evidenciadas por la aplicación de la prueba estadística ANOVA de un factor. Conclusión. Se construyó un modelo técnico estructural por fases, describiendo 3 componentes, 3 periodos y 11 fases para cada rol, el cual resulta ser más integral permitiendo apuntar a la optimización del rendimiento deportivo y a la mejora de los resultados tanto indivuales como en la competición por parejas.

Palabras clave: Toss to hands, cheerleaders, fases de movimiento, base, flayer.

\section{Introducción}

\subsection{Un acercamiento a las generalidades}

El cheerleader es considerada una actividad deportiva de carácter artístico, que fundamenta sus técnicas basado en elementos de otros deportes como la gimnasia artística, aeróbica y rítmica, acompañados de la danza y manejo del ritmo Stroescu, (2018); Artemyeva y Moshenska, (2018), lyubimova, (2019); así como elementos del levantamiento olímpico de pesas, deporte con estatus olímpico caracterizado por su explosión y potencia (Leitz, 2015). 
La estructura competitiva de esta actividad deportiva se presenta a través de rutinas de coreografías Li y Chen, (2017), con elementos acrobáticos individuales y en parejas con ejercicios que requieren principalmente una postura adecuada, equilibrio estático, dinámica motriz y una elegancia en la ejecución de cada movimiento (Bilenka, 2017).

Una de las técnicas más utilizadas para la realización de coreografías y secuencias grupales es el Toss to Hands Eckley, (2018), considerada como una técnica combinada de trabajo en pareja. En la ejecución técnica actúa un atleta denominado "base", que ejecuta las acciones de potencia con movimientos similares a la técnica de la cargada olímpica Zdunek, (2020) y un atleta que actúa como flayer con acciones gimnastica acrobáticas de vuelo con una posterior mantención de postura (Smith, 2017).

Las ciencias aplicadas al deporte como la fisiología del ejercicio, la medicina deportiva, la biomecánica deportiva, por nombrar algunas, rara vez se aplican en esta disciplina deportiva de manera integrada Becker \& Wu, (2015), tomando en cuenta que los elementos a demostrar son rápidos, similares al trabajo de fuerza explosiva en la halterofilia, por tal razón se desarrollara una base de distintos estudios para determinar una secuencia y patrones biomecánicos de ejecución técnica que pueden extrapolar al cheerleaders Verheul et al., (2020). En su mayoría los levantadores de chicas, las bases ejecutan distintos ejercicios para desarrollar la habilidad de poder levantar a su pareja en el Toss to hands, las rutinas de cheerleaders exigen un alto nivel de fuerza explosiva de tren inferior.

La Biomecánica es la ciencia aplicada al deporte que nos permite comprender la cinética (fuerzas), cinemática (movimientos) del cuerpo y su interacción con respecto a los desplazamientos, el equilibrio estático y la actividad dinámica que caracteriza a las acciones deportivas gimnasticas y acrobáticas (Nyman, 2020).

Dentro del cheerleaders los pocos estudios biomecánicos han pretendido evaluar sus fases de movimiento y caracterizar ciertos parámetros biomecánicos como la fuerza aplicada al momento del lanzamiento Eckley, (2018), cinemática de los lanzamientos en acciones como pre-extensión Carrillo y Herrera, (2020), o estudios de la biomecánica del calzado utilizado por los atletas en la ejecución de las diferentes técnicas Johnsom, (2020), pero no se han encontrado evidencias de trabajos investigativos sobre la biomecánica de la ejecución de la técnica de Toss to hands.

Para muchos deportistas y entrenadores, es difícil comprender los errores técnicos de diferentes acciones deportivas que caracterizan sus actividades, sin poder llegar a la optimización de un movimiento, el cual se puede lograr con la ayuda de un análisis de tipo cualitativo para describir la técnica en diferentes fases y/o cuantitativo para analizar esta técnica en base a diferentes parámetros numéricos (Colyer et al., 2018, Sierra et al, 2020;).

En el cheerleaders más aun cuando las bases realizan acciones del toss to hands, a la vista del ser humano muchos movimientos rápidos e involuntarios pueden desfavorecer la ejecución de distintas técnicas que se pretenden mejorar, la importancia del control biomecánico a través de una observación indirecta aplicando un análisis videográfico, 
puede facilitar la correcta ejecución de técnicas y movimientos innecesarios Medina, Toledo y Sánchez, (2020), dicho proceso se aplica en la actualidad con programas especializados de análisis, que pueden desacelerar los movimientos y a la vista de entrenadores, deportistas y estudiantes, sea más fácil entender los errores técnicos cometidos en la ejecución técnica para un proceso posterior de corrección y perfeccionamiento Sánchez, (2018), Penichet-Tomás et al, (2019)

Por tal razón es necesario desarrollar un estudio sobre la aplicación del análisis biomecánico de la ejecución técnica del Toss to hands en el cheerleaders, planteando un desglose de los parámetros cinéticos y cinemáticos que los caracteriza. La información permitirá encaminar el proceso de entrenamiento deportivo bajo un control directo del deportista sobre el conocimiento de las fases del movimiento, velocidades de ejecución y percepción de momentos angulares, el entrenador de su parte podrá diseñar un entrenamiento mucho más inteligente encaminado no solo al desarrollo de capacidades físicas determinantes y condicionantes para esta actividad deportiva, sino bajo un proceso de corrección de errores técnicos y de esta manera elevar la eficiencia y eficacia de la preparación y un resultado deportivo.

Como objetivo principal del estudio se plantea analizar biomecánicamente la ejecución técnica del Toss To Hands en cheerleaders determinando sus fases de movimiento a través de la construcción de un modelo técnico estructural y el análisis de diferentes parámetros como los valores angulares de las articulaciones del codo, cadera y rodilla y sus variaciones al igual que la velocidad y altura máxima en cada fase.

La particularidad de esta nueva disciplina deportiva del cheerleaders en nuestro país, exige a los atletas un nivel elevado del rendimiento técnico, que permita demostrar con satisfacción las rutinas en excelente nivel, considerando la exclusiva del toss to hands, demostrando un rendimiento óptimo con resultados.

Es pertinente analizar las características biomecánicas de la ejecución técnica del toss to hands en el cheerleaders para determinar los fundamentos cinéticos y cinemáticos como un paso fundamental para mejorar la probabilidad de lograr una mayor precisión para varias tareas importantes, es decir, resumen de video, eventos clave selección, y para suprimir las tasas de clasificación errónea (Minhas et al., 2019).

\section{Metodología}

El trabajo de investigación desarrollado siguió un enfoque mixto, de tipo descriptivo con un corte transversal. Estando acompañado de la utilización de los métodos teóricos tales como: el histórico- lógico, analítico- sintético, el inductivo-analítico y el sistémico estructural -funcional los cuales permitieron analizar los antecedentes teóricos del tema y favorecieron la construcción del modelo estructural por fases para la ejecución de la técnica en estudio y a su vez determinar las características biomecánicas que definen la ejecución de la técnica.

La población de objeto de estudio estuvo representada por los atletas que practican la disciplina de Cheerleader en la provincia de Pichincha - Ecuador, la cual fue seleccionada 
aplicando un muestreo probabilístico por conveniencia, quedando integrada la muestra por 25 atletas que representan a un club deportivo formativo de la ciudad de Quito.

La técnica aplicada fue la observación a través del análisis biomecánico de la ejecución de la técnica de Toss to hands, como instrumento se utilizó el software libre para análisis de parámetros biomecánicos KINOVEA versión 0.8.27. En el proceso de análisis biomecánico se determinaron los siguientes parámetros de estudio: ángulos de la articulación de codo, cadera y rodilla; alturas y la velocidad de ejecución en las diferentes fases del movimiento, tanto en los atletas que ejecutan el rol de base como los que ejecutan el rol de flayer.

Para la captura de los movimientos de ejecución técnica con el objetivo de unificar los protocolos para la realización de los videos de análisis, los entrenadores de los deportistas utilizaron la misma cámara de video con las siguientes características: cámara digital dual de 12 Mpx con gran angular: apertura de f/1,8; Teleobjetivo: apertura de $f / 2,8$ Zoom óptico x2; zoom digital hasta x10; Velocidad de grabación de vídeo en 720p HD a 60 fps en cámara lenta.

Cabe destacar que para dar cumplimiento a los objetivos planteados en la investigación, en primer lugar, se analizó los diferentes videos realizados, para construir el modelo técnico estructural de la técnica por fases, determinando los componentes, periodos y fases de ejecución de la técnica del Toss to hands. Posterior a esto se realizó un análisis individual del video de cada atleta, diferenciando el rol que estos desempeñan en la ejecución de la técnica en estudio, para determinar los valores de los parámetros biomecánicos planteados en la metodología de investigación.

El procesamiento estadístico de los datos obtenidos en la investigación, se realizó aplicando el paquete estadístico SPSS versión 25, efectuando un análisis descriptivo que determinó los valores mínimos, máximos, medios y sus respectivas desviaciones estándares para las variables cuantitativas y un análisis de frecuencias y porcentajes para las variables cualitativas que caracterizaron a la muestra de estudio. Para el análisis general de los resultados se aplicó una prueba de normalidad de Shapiro-will que determinó la aplicación de una prueba paramétrica ANOVA de un factor, para determinar las diferencias significativas entre los parámetros biomecánicos en las diferentes fases del movimiento.

\section{Resultados}

En primer lugar, se realizó el proceso de caracterización de la muestra de estudio según los siguientes parámetros (Tabla No. 1)

Tabla No 1: Características de la muestra de estudio.

\begin{tabular}{|c|c|c|c|c|c|c|c|}
\hline Género & Rol & $\begin{array}{c}\text { Parámetro de } \\
\text { caracterización }\end{array}$ & $\mathrm{n}$ & Mínimo & Máximo & Media & $\begin{array}{c}\text { Desviación } \\
\text { estándar }\end{array}$ \\
\hline \multirow{2}{*}{$\begin{array}{c}\text { Masculino } \mathrm{n} \\
\text { (13) } 52 \%\end{array}$} & \multirow{2}{*}{ Base } & Edad & & 15 & 20 & 16.92 & \pm 1.55 \\
\hline & & Peso & 13 & 41 & 104 & 62.85 & \pm 18.33 \\
\hline
\end{tabular}


ISSN: 2602-8085

\begin{tabular}{|c|c|c|c|c|c|c|c|}
\hline & & Talla & & 1.60 & 1.85 & 1.68 & \pm 0.07 \\
\hline \multirow{6}{*}{$\begin{array}{l}\text { Femenino n } \\
\text { (12) } 48 \%\end{array}$} & \multirow{3}{*}{ Base } & Edad & \multirow{3}{*}{2} & 15 & 16 & 15.50 & \pm 0.71 \\
\hline & & Peso & & 55 & 62 & 58.50 & \pm 4.95 \\
\hline & & Talla & & 1.55 & 1.63 & 1.59 & \pm 0.06 \\
\hline & \multirow{3}{*}{ Flayer } & Edad & & 13 & 17 & 15.00 & \pm 1.25 \\
\hline & & Peso & 10 & 40 & 60 & 47.80 & \pm 6.96 \\
\hline & & Talla & & 1.48 & 1.64 & 1.54 & \pm 0.05 \\
\hline \multicolumn{3}{|c|}{ TOTAL } & \multicolumn{5}{|c|}{25} \\
\hline
\end{tabular}

Fuente: Elaboration propia

El proceso de observación de los videos realizados y la fundamentación teórica del estudio permitió construir el modelo técnico estructural por fases de la ejecución de la técnica Toss to hands, determinando los componentes del movimiento, los periodos, sus respectivas fases y acciones motrices, diferenciando el rol de cada atleta en su trabajo de pareja (Tabla No. 2).

Tabla No. 2: Modelo estructural de la técnica por fases de la ejecución de la técnica Toss to hands

\begin{tabular}{|c|c|c|c|c|c|}
\hline ROL & COMPONENTES & PERIODOS & No. & FASES & ACCIONES \\
\hline \multirow{11}{*}{ BASE } & \multirow{4}{*}{ Preparatorio } & \multirow{4}{*}{ Agarre } & 1 & $\begin{array}{l}\text { Posición } \\
\text { inicial }\end{array}$ & $\begin{array}{l}\text { Flexión de rodillas, cadera y } \\
\text { agarre de cadera de la flayer. }\end{array}$ \\
\hline & & & 2 & Activación & $\begin{array}{l}\text { Flexión de rodillas, cadera } \\
\text { y flexión de codos. }\end{array}$ \\
\hline & & & 3 & $\begin{array}{l}\text { Primer } \\
\text { impulso }\end{array}$ & $\begin{array}{l}\text { Extensión de rodillas, cadera } \\
\text { y flexión de codos. }\end{array}$ \\
\hline & & & 4 & $\begin{array}{l}\text { Segundo } \\
\text { impulso }\end{array}$ & $\begin{array}{l}\text { Flexión máxima de rodillas, } \\
\text { cadera, semi flexión de } \\
\text { brazos. }\end{array}$ \\
\hline & \multirow{3}{*}{ Principal } & \multirow{3}{*}{ Elevación } & 5 & Proyección & $\begin{array}{l}\text { Extensión total de piernas en } \\
\text { la articulación de rodilla, } \\
\text { cadera y extensión total de } \\
\text { brazos. }\end{array}$ \\
\hline & & & 6 & Agarre & $\begin{array}{l}\text { Rotación de hombros, } \\
\text { flexión pierna de equilibrio, } \\
\text { extensión de cadera y } \\
\text { flexión de codos. }\end{array}$ \\
\hline & & & 7 & Estabilización & $\begin{array}{l}\text { Extensión de rodilla, cadera } \\
\text { y flexión de codos. }\end{array}$ \\
\hline & \multirow{4}{*}{ Conclusivo } & \multirow{4}{*}{ Desmonte } & 8 & $\begin{array}{l}\text { Impulso de } \\
\text { desmonte }\end{array}$ & $\begin{array}{l}\text { Flexión de rodillas, cadera } \\
\text { y flexión de codos. }\end{array}$ \\
\hline & & & 9 & Desmonte & $\begin{array}{l}\text { Agarre de cadera, extensión } \\
\text { de rodillas, cadera y codos. }\end{array}$ \\
\hline & & & 10 & Frenado & $\begin{array}{l}\text { Flexión de rodillas, cadera y } \\
\text { codos. }\end{array}$ \\
\hline & & & 11 & Posición final & $\begin{array}{l}\text { Extensión de todas las } \\
\text { articulaciones. }\end{array}$ \\
\hline ROL & COMPONENTES & PERIODOS & & FASES & ACCIONES \\
\hline \multirow{3}{*}{ FLAYER } & \multirow{3}{*}{ Preparatorio } & \multirow{3}{*}{ Agarre } & 1 & $\begin{array}{l}\text { Posición } \\
\text { inicial }\end{array}$ & $\begin{array}{l}\text { Flexión de codos y agarre de } \\
\text { muñecas de la base. }\end{array}$ \\
\hline & & & 2 & Activación & $\begin{array}{l}\text { Flexión de rodillas, cadera y } \\
\text { codos. }\end{array}$ \\
\hline & & & 3 & $\begin{array}{l}\text { Primer } \\
\text { impulso }\end{array}$ & $\begin{array}{l}\text { Extensión de rodillas, cadera } \\
\text { y flexión de codos. }\end{array}$ \\
\hline
\end{tabular}




$\begin{array}{cccc} & 4 & \begin{array}{l}\text { Segundo } \\ \text { impulso }\end{array} & \begin{array}{l}\text { Flexión máxima de rodillas, } \\ \text { cadera y codos. } \\ \text { Extensión total de piernas en } \\ \text { la articulación de rodillas y } \\ \text { cadera. } \\ \text { Principal }\end{array} \quad \text { Elevación } \\ \text { cadera. }\end{array}$

Fuente: Elaboración propia

El modelo estructural se construyó basado en 3 componentes cada uno con 3 periodos y 11 fases de movimiento, fases muy similares entre las acciones que realiza la base y el o la flayer, con la existencia de una fase de vuelo en las acciones realizadas por este último para poder realizar la postura estática sobre las manos de la base (Figura No. 1).

Figura No 1: Kinograma de la ejecución de la técnica Toss to hands

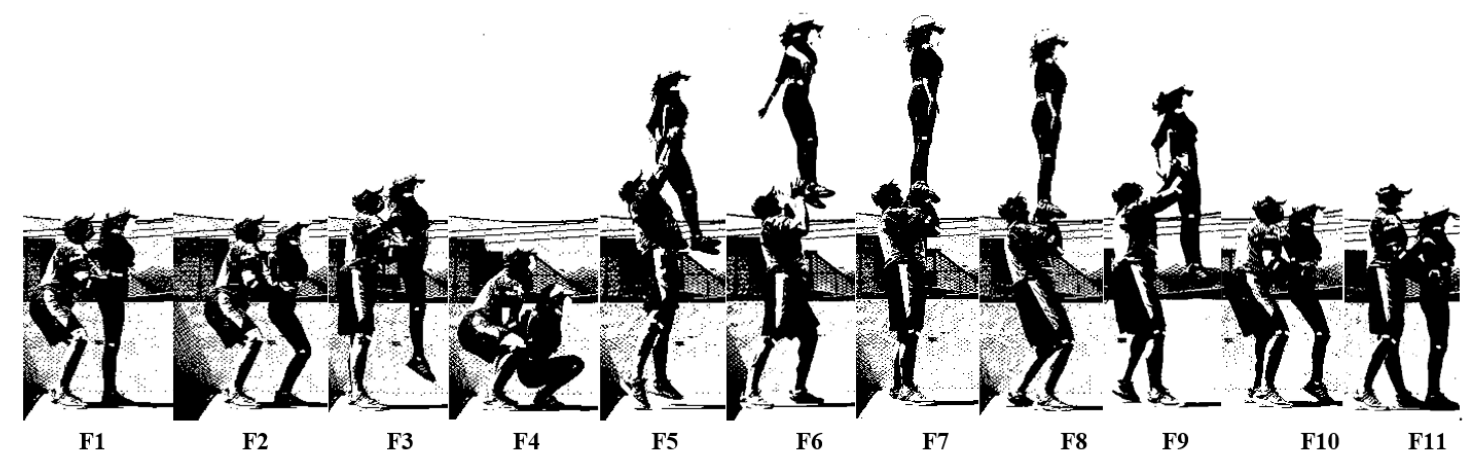

Fuente: Elaboración propia

Al establecer el modelo técnico estructural por fases de la técnica, se pudo realizar el análisis biomecánico de los diferentes parámetros planteados en la metodología preliminar de estudio, observando la diferencia entre los valores angulares, de velocidad y alturas en las diferentes fases del movimiento. El análisis de cada integrante de la muestra de estudio en sus diferentes roles, permitió determinar los valores medios que caracterizan a cada fase según el parámetro analizado (Tabla No. 3).

Tabla No. 3: Parámetros biomecánicos de la ejecución de la técnica Toss to hands 
ISSN: 2602-8085

Vol. 5, N³, p. 36-47, julio-septiembre, 2021

\begin{tabular}{|c|c|c|c|c|c|c|}
\hline Rol & $\begin{array}{c}\text { FASE } \\
\text { No. }\end{array}$ & $\begin{array}{c}\text { Ángulo } \\
\text { articulación } \\
\text { de la rodilla }\end{array}$ & $\begin{array}{c}\text { Ángulo } \\
\text { articulación } \\
\text { de la cadera }\end{array}$ & $\begin{array}{c}\text { Ángulo } \\
\text { articulación } \\
\text { del codo }\end{array}$ & Velocidad & $\begin{array}{l}\text { Altura } \\
\text { máxima }\end{array}$ \\
\hline \multirow{11}{*}{ Base } & 1 & $121^{\circ}$ & $113^{\circ}$ & $103^{\circ}$ & $0 \mathrm{~m} / \mathrm{s}$ & $148,03 \mathrm{~cm}$ \\
\hline & 2 & $123^{\circ}$ & $108^{\circ}$ & $112^{\circ}$ & $0.084 \mathrm{~m} / \mathrm{s}$ & $149,62 \mathrm{~cm}$ \\
\hline & 3 & $164^{\circ}$ & $155^{\circ}$ & $95^{\circ}$ & $0.164 \mathrm{~m} / \mathrm{s}$ & $170,30 \mathrm{~cm}$ \\
\hline & 4 & $105^{\circ}$ & $77^{\circ}$ & $120^{\circ}$ & $0.110 \mathrm{~m} / \mathrm{s}$ & $125,53 \mathrm{~cm}$ \\
\hline & 5 & $164^{\circ}$ & $199^{\circ}$ & $160^{\circ}$ & $0.405 \mathrm{~m} / \mathrm{s}$ & $216,46 \mathrm{~cm}$ \\
\hline & 6 & $162^{\circ}$ & $180^{\circ}$ & $60^{\circ}$ & $0.150 \mathrm{~m} / \mathrm{s}$ & $157,45 \mathrm{~cm}$ \\
\hline & 7 & $170^{\circ}$ & $175^{\circ}$ & $37^{\circ}$ & $0.031 \mathrm{~m} / \mathrm{s}$ & $157,59 \mathrm{~cm}$ \\
\hline & 8 & $134^{\circ}$ & $152^{\circ}$ & $37^{\circ}$ & $0.033 \mathrm{~m} / \mathrm{s}$ & $148,03 \mathrm{~cm}$ \\
\hline & 9 & $163^{\circ}$ & $160^{\circ}$ & $120^{\circ}$ & $0.812 \mathrm{~m} / \mathrm{s}$ & $185,98 \mathrm{~cm}$ \\
\hline & 10 & $119^{\circ}$ & $121^{\circ}$ & $102^{\circ}$ & $0.316 \mathrm{~m} / \mathrm{s}$ & $161,58 \mathrm{~cm}$ \\
\hline & 11 & $179^{\circ}$ & $178^{\circ}$ & $180^{\circ}$ & $0 \mathrm{~m} / \mathrm{s}$ & $172,58 \mathrm{~cm}$ \\
\hline \multicolumn{2}{|c|}{$\mathrm{P}$ valor } & $0.00 *$ & $0.02 *$ & $0.03 *$ & $0.05 *$ & $0.05^{*}$ \\
\hline Rol & $\begin{array}{c}\text { FASE } \\
\text { No. }\end{array}$ & $\begin{array}{c}\text { Ángulo } \\
\text { articulación } \\
\text { de la rodilla }\end{array}$ & $\begin{array}{c}\text { Ángulo } \\
\text { articulación } \\
\text { de la cadera }\end{array}$ & $\begin{array}{l}\text { Ángulo } \\
\text { articulación } \\
\text { del codo }\end{array}$ & Velocidad & $\begin{array}{l}\text { Altura } \\
\text { máxima }\end{array}$ \\
\hline \multirow{11}{*}{ Flayer } & 1 & $179^{\circ}$ & $178^{\circ}$ & $116^{\circ}$ & $0 \mathrm{~m} / \mathrm{s}$ & $96,17 \mathrm{~cm}$ \\
\hline & 2 & $134^{\circ}$ & $149^{\circ}$ & $113^{\circ}$ & $0.042 \mathrm{~m} / \mathrm{s}$ & $147,21 \mathrm{~cm}$ \\
\hline & 3 & $184^{\circ}$ & $186^{\circ}$ & $120^{\circ}$ & $1.037 \mathrm{~m} / \mathrm{s}$ & $124,66 \mathrm{~cm}$ \\
\hline & 4 & $43^{\circ}$ & $64^{\circ}$ & $127^{\circ}$ & $0.152 \mathrm{~m} / \mathrm{s}$ & $95,48 \mathrm{~cm}$ \\
\hline & 5 & $167^{\circ}$ & $160^{\circ}$ & $180^{\circ}$ & $2.498 \mathrm{~m} / \mathrm{s}$ & $257,85 \mathrm{~cm}$ \\
\hline & 6 & $182^{\circ}$ & $179^{\circ}$ & $180^{\circ}$ & $0.435 \mathrm{~m} / \mathrm{s}$ & $303,14 \mathrm{~cm}$ \\
\hline & 7 & $180^{\circ}$ & $189^{\circ}$ & $180^{\circ}$ & $0.080 \mathrm{~m} / \mathrm{s}$ & $296,13 \mathrm{~cm}$ \\
\hline & 8 & $174^{\circ}$ & $180^{\circ}$ & $180^{\circ}$ & $0.232 \mathrm{~m} / \mathrm{s}$ & $282,30 \mathrm{~cm}$ \\
\hline & 9 & $180^{\circ}$ & $184^{\circ}$ & $173^{\circ}$ & $3.902 \mathrm{~m} / \mathrm{s}$ & $248,11 \mathrm{~cm}$ \\
\hline & 10 & $152^{\circ}$ & $157^{\circ}$ & $147^{\circ}$ & $2.452 \mathrm{~m} / \mathrm{s}$ & $161.45 \mathrm{~cm}$ \\
\hline & 11 & $178^{\circ}$ & $179^{\circ}$ & $179^{\circ}$ & $0 \mathrm{~m} / \mathrm{s}$ & $152,01 \mathrm{~cm}$ \\
\hline \multicolumn{2}{|c|}{$\mathrm{P}$ valor } & $0.03^{*}$ & $0.05^{*}$ & $0.05^{*}$ & $0.00 *$ & $0.04 *$ \\
\hline
\end{tabular}

El análisis de los parámetros biomecánicos establecidos permitió determinar que las mayores velocidades que llega a presentar la base, las cuales se desarrollan en la fase 9 de desmonte y la fase 5 de proyección, fenómeno que se presenta de igual manera en las mismas en las acciones ejecutadas por el o la flayer, justificando esta aceleración por el efecto de la fuerza que se produce por las diferentes acciones de agarre en estas fases, resultados estadísticamente diferentes en cada fase del movimiento.

Como puede apreciarse los valores angulares de las articulaciones del codo, cadera y rodilla son diferentes estadísticamente en cada fase del movimiento, dependiendo de las acciones de flexión o extensión necesarias para el desarrollo de los niveles óptimos de velocidad, producto de la aplicación de fuerzas de agarre en las diferentes fases. En relación a la altura máxima los valores establecidos dependen de la estatura tanto de la base y del o la flayer y la correcta postura de cada uno de ellos para la ejecución de las fases dinámicas y estáticas de esta técnica. 
Dando continuidad al análisis realizado es importante destacar que el trabajo de investigación desarrollado se puede considerar como pionero en el contexto ecuatoriano, en esta disciplina deportiva y de animación, debido a que independientemente de todos los avances logrados aún son escasos los estudios científicos realizados sobre las técnicas de ejecución de las diferentes acciones que se pueden realizar de manera individual o en pareja.

Precisando los resultados obtenidos es significativo plantear que el estudio que se presenta aporta un modelo estructural de la técnica de ejecución denominada Toss to hands, técnica esta que posee 3 componentes, 3 periodos y 11 fases de movimiento, tanto para los roles de base como de flayer, coincidiendose con las investigaciones realizadas por Moreno (2018), el cual planteo una estructura de la técnica con un elemento similar a la técnica del Toss to hands, con la particularidad de una fase de vuelo sin apoyo considerado como lanzamiento, presentando una fase de preparación, impulso y despegue, vuelo, ejecución o punto cero, recuperación, recepción y termino fases comunes para ambos roles, con especificidades de las acciones motrices de cada uno, fenómeno que se asemejan a nuestra construcción. De igual manera Carrillo y Herrera (2020), plantean que este tipo de técnicas de trabajo en parejas se caracterizan por tener la ejecución a través de una fase de impulsión, de propulsión, mantenimiento, expulsión y fase de recepción, siendo más concreto y sintéticos en las acciones que ambos roles ejecutan, a diferencia de nuestro estudio que plantea un estudio más profundo en la definición de las fases.

De igual manera Moreno (2018) realiza un análisis angular de la técnica tomando en cuenta además a la articulación del hombro y tobillo, no analiza la velocidad de cada fase, sino la velocidad angular en cada articulación.

Todo lo señalado demuestra la valía del trabajo realizado; el modelo estructural aportado basado en la técnica de ejecución denominada Toss to hands, dada sus propias características al identificar en esta técnica 3 componentes, 3 periodos y 11 fases de movimiento, tanto para los roles de base como de flayer, resulta ser más integral y permite apuntar a la optimización del rendimiento deportivo y a la mejora de los resultados tanto indivuales como en la competición por parejas.

\section{Conclusiones}

- El estudio permitió construir un modelo técnico estructural por fases de la ejecución de la técnica de Toss to hands en el cheerleaders, describiendo 3 componentes, 3 periodos y 11 fases para cada rol dentro de la técnica, el flayer a excepción de la base presenta una fase de vuelo y una fase de equilibrio estático y los valores de velocidad más altos se presentan en este último sobre todo en la fase de desmonte, por la acción de la fuerza externa aplicada por la base en el momento del agarre, el cual resulta ser más integral permitiendo apuntar a la optimización del rendimiento deportivo y a la mejora de los resultados tanto 
indivuales como en la competición por parejas, denotándose su pertinencia y actualidad.

\section{Referencias bibliográficas}

Artemyeva, G., \& Moshenska, T., (2018). Role and importance of choreography in gymnastic and dance sports. Slobozhanskyi herald of science and sport, (4 (66)), 27-30.

Becker, J., \& Wu, W. F. W. (2015). Integrating biomechanical and motor control principles in elite high jumpers: A transdisciplinary approach to enhancing sport performance. Journal of Sport and Health Science, 4(4), 341-346. https://doi.org/10.1016/j.jshs.2015.09.004

Bilenka, I.G. (2017), Muzychno-rutmichne vyhovanie u vydah sportu estetuchnoi spriamovanosti [Musical-rhythmic upbringing in the sports of aesthetic orientation], KSAPC, Kharkiv. (in Ukr.)

Carrillo Gómez, D. N., \& Herrera Cubillos, J. A. (2020). Análisis cinemático del gesto técnico lanzamiento a pre-extensión en cheerleaders de fusagasuga (Doctoral dissertation).

Colyer, S. L., Evans, M., Cosker, D. P., \& Salo, A. I. T. (2018). A Review of the Evolution of Vision-Based Motion Analysis and the Integration of Advanced Computer Vision Methods Towards Developing a Markerless System.

Eckley, K. (2018). The Physics of Cheerleading: Force Production of Cheerleading Stunts.

Johnson, A. C. (2020). Biomechanical Comparison of" Old" and" New" Cheer Shoes in Collegiate Cheerleaders.

Leitz, R. S. (2015). The Relationship Between Core Stability Related Measures and Performance in Adolescent All-Star Cheerleaders.

Li, X., \& Chen, L. (2017). Under the Old Rules and New Alternate Study on Cheerleading Dance Choreography. Sichuan Sports Science, 03.

Lyubimova-Lisa, M. (2019). Cheerleading: Interaction of sport an choreography. Intellectual Economics, Management and Education, 350.

Medina Cabrera, M. L., Toledo Ríos, R., \& Sánchez Oms, A. B. (2020). Procedimiento para el análisis biomecánico de la variabilidad del movimiento en el lanzamiento de disco. 
Minhas, R. A., Javed, A., Irtaza, A., Mahmood, M. T., \& Joo, Y. B. (2019). Shot classification of field sports videos using AlexNet Convolutional Neural Network. Applied Sciences (Switzerland), 9(3). https://doi.org/10.3390/app9030483

Nyman, E. (2020). Biomechanics of Gymnastics. In Gymnastics Medicine (pp. 27-54). Springer, Cham.

Nor Adnan, N. M., Ab Patar, M. N. A., Lee, H., Yamamoto, S. I., Jong-Young, L., \& Mahmud, J. (2018). Biomechanical analysis using Kinovea for sports application. IOP Conference Series: Materials Science and Engineering, 342(1). https://doi.org/10.1088/1757-899X/342/1/012097

Penichet-Tomás, A., Jimenez-Olmedo, J. M., Sebastiá-Amat, S., \& Pueo, B. (2019). Mejora de la técnica de remo mediante la utilización de análisis de vídeo en el Grado de Ciencias del Deporte.

Sanchez, A. (2018). El uso del kinovea para el analisis biomecanico desde uns perspectiva cuantitativa. TRANCES Revista de Transmisión Del Conocimiento Educativo y de La Salud., 10(6), 725-738.

Sierra, A. J. P., Valdez, A. E. G., Sainz, H. M. S., Orozco, S. I. V., \& Reyes, F. B. Proceso de corrección en errores técnicos para los atletas en formación. 2, 3 y 4 de diciembre de 2020 Modalidad Virtual.

Smith, N. S. (2017). A Comparison of Physiologically-Based Pharmacokinetic (PBPK) Models of Methyl-Tertiary Butyl Ether (MTBE).

Stroescu, A. (2018). Operative syntheses on cheerleading training. Journal of Sport and Kinetic Movement, 2(31), 18-23.

Verheul, J., Nedergaard, N. J., Vanrenterghem, J., \& Robinson, M. A. (2020). Measuring biomechanical loads in team sports-from lab to field. Science and Medicine in Football. https://doi.org/10.1080/24733938.2019.1709654

Zdunek, A. (2020). Who Knows the Difference Between Competitive Cheerleading, Sideline Cheerleading, Acrobatics and Tumbling? Why this Distinction is so Important for Title IX. Marquette Sports Law Review, 31(1), 175.

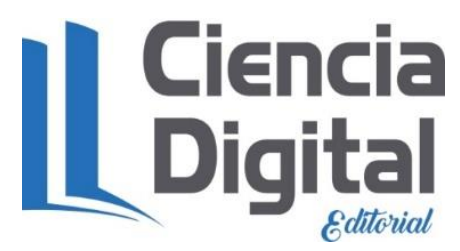


PARA CITAR EL ARTÍCULO INDEXADO.

Orbe Yumisaca, O. A. (2021). Análisis biomecánico de la ejecución técnica del Toss to hands en el Cheerleaders. Ciencia Digital, 5(3), 36-47. https://doi.org/10.33262/cienciadigital.v5i3.1731

\section{Ciencia \\ LDigital}

El artículo que se publica es de exclusiva responsabilidad de los autores y no necesariamente reflejan el pensamiento de la Revista Ciencia Digital.

El artículo queda en propiedad de la revista y, por tanto, su publicación parcial y/o total en otro medio tiene que ser autorizado por el director de la Revista Ciencia Digital.
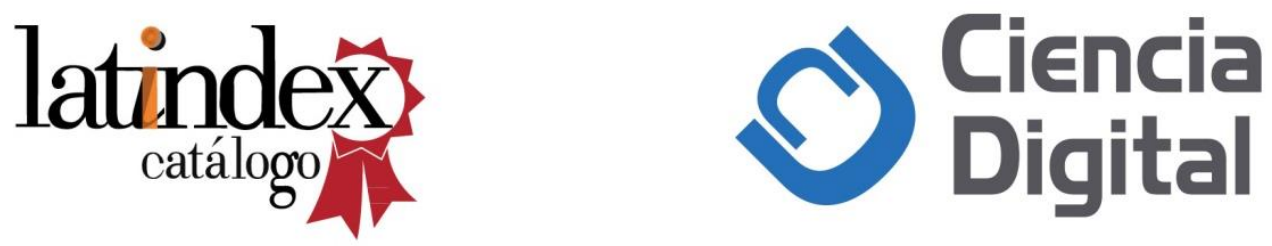